\title{
ECONOMIC ANALYSIS ENZYMATIC DEBRIDEMENT VS. STANDARD OF CARE IN BURN TREATMENT PRELIMINARY DATA
}

\author{
J. MINIC, E.VIGATO, M. GOVERNA \\ Azienda Ospedaliera Universitaria Integrata VERONA
}

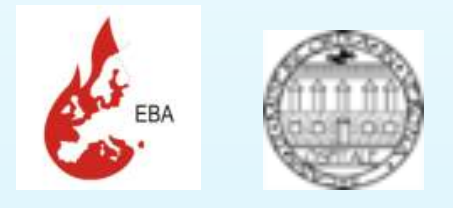

Cost analysis of the burn care is a well known challenge due to the heterogeneity of the patients, injuries and treatment strategies well as the large number of specialties of highly trained burn care personal involved and the extensive resources needed. The issue is complicated by the different national, regional and even organizational reimbursement modalities. The aim of this study was to assess as much realistically as possible the real costs of the burn care, issue by issue, in our Burn Center.

\section{MATERIAL AND METHOD}

Retrospective analysis of 44 patients divided in two equal groups; the standard of care group (SOC) and a minimal invasive modality (MIM)based on NexoBrid $®$ (NXB) enzymatic debridement ( ED ) group. Same inclusion/exclusion criteria were used for both cohort groups. All the patients had deep partial and full thickness burns. We compared different medically and economically important factors and measures as cost-effectiveness parameters: Days of hospitalization, time to complete debridement, place of debridement performing ( BU or ICU or OT), human resources, devices, medication and disposables consuming, blood consuming.

Statistical analysis: Chi - square test; Wilcoxon- Mann- Whitney test

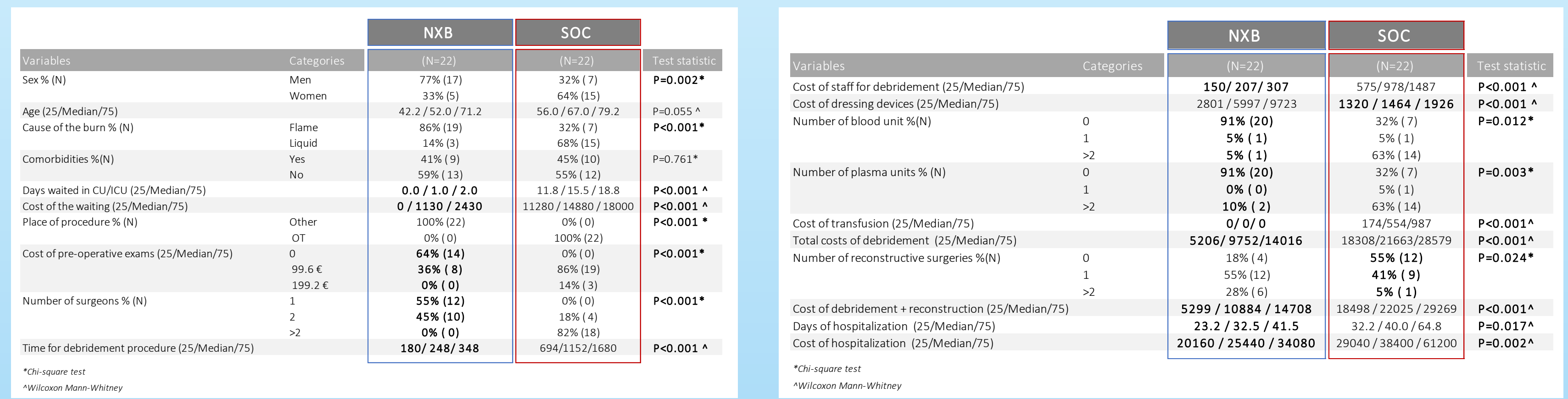

RESULTS: TIME TO DEBRIDMENT , SURGICAL BURDEN \& \& COSTS
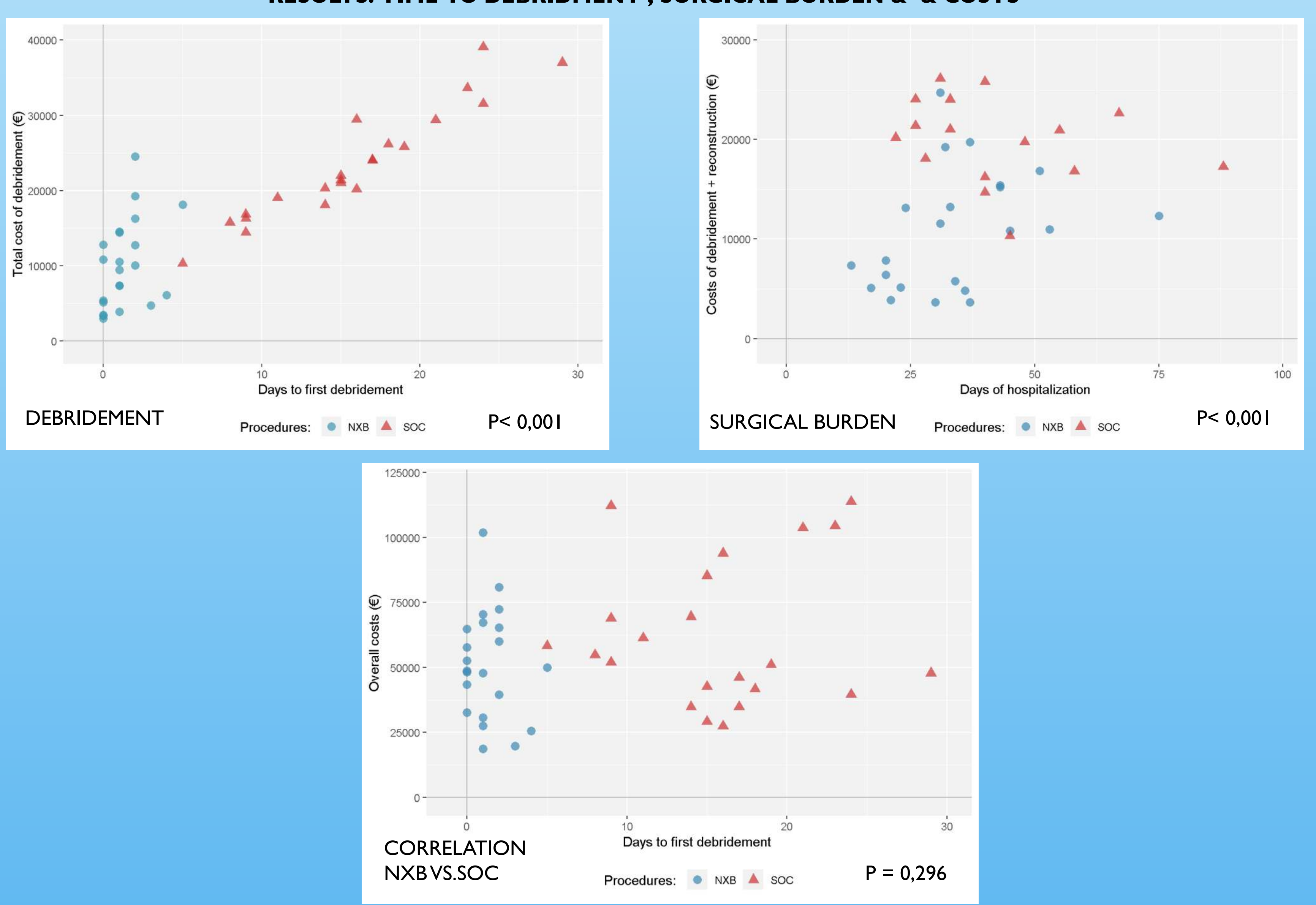

\section{CONCLUSION}

This study demonstrated a clear economical advantage ED vs SOC in reducing hospitalization ( 749 days vs. 1068 days ), surgical burden and costs of $2310 €$ vs $12705,6 €$, average total saving per patient NXB vs SOC of II $228 €$.

The study is limited by the small number of cases and its validity on Italian health care system.

The real measure to weigh the merits of NXB MIM compared to SOC will be the final out come of function, cosmesis and quality of life two years post injury that should be at least as good as the SOC. 